\title{
Crystal structure of benzyl (2,6-diisocyano-4-methyl)phenyl ether, $\mathrm{C}_{16} \mathrm{H}_{12} \mathrm{~N}_{2} \mathrm{O}$
}

\author{
F. E. Hahn, P. Hein and T. Lügger
}

Freie Universität Berlin. Institut für Anorganische und Analytische Chemie, Fabeckstrasse 34-36, D-12305 Berlin, Germany

Received November 23, 1997, CSD-No. 409168

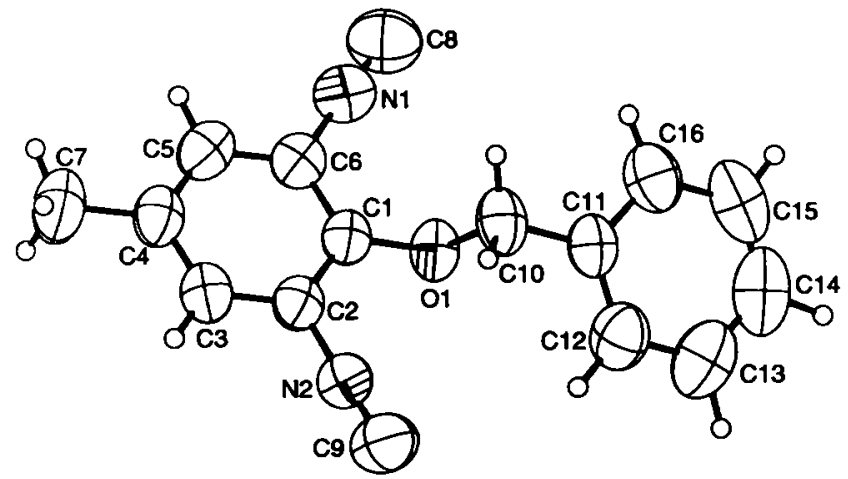

Source of material: Crystals of the title compound were obtained by recrystallization from $\mathrm{CH}_{2} \mathrm{Cl}_{2} /$ hexane. The title compound was synthezised by the following sequence: reaction of 4methylhydroxybenzene with fuming nitric acid, reduction of the formed 2,5-dinitro-4-methylhydroxybenzene with hydrazine, conversion of the primary amines into formamide functions by refluxing with phenylformiate in ethanol, protection of the hydroxyl function with benzylbromide and finally conversion of the formamide functions into isocyanides with diphosgene according to the method of Ugi (see ref. 1). The isocyanide formation is not possible with the free hydroxy compound. The overall yield is $25 \%$ based on 4-methylhydroxybenzene.

We reported recently on an effective way for the synthesis of $\mathrm{N}, \mathrm{O}$-heterocarbene complexes using the intramolecular nucleophilic attack of the hydroxy group of coordinated 2-hydroxyphenylisocyanide (see ref. 2). This reaction is reversible and the reaction product depends on the electronic situation at the metal center (see ref. 3). The title compound features two isocyanides, which upon coordination to different metals will compete for the hydroxyl group liberated after $\boldsymbol{O}$-benzyl bond cleavage. It will therefore allow for a direct comparison of the carbene formation tendency of two different metal centers under otherwise completely identical reaction conditions. Bond lengths and angles in the compound are normal. The $\mathrm{N} \equiv \mathrm{C}$ separations in the isocyanide groups of 1.141 (3) $\AA$ and $1.145 \AA$ fall in the range observed previously for functionalized arylisocyanides (see refs. 4-6).

$\mathrm{C}_{16} \mathrm{H}_{12} \mathrm{~N}_{2} \mathrm{O}$, monoclinic, $P 12_{1} / c 1$ (No. 14), $a=4.238(4) \AA$, $b=12.276(2) \AA, c=26.218(9) \AA, \beta=93.84(5)^{\circ}, V=1360.9 \AA^{3}$, $Z=4, \rho_{\mathrm{m}}=1.218 \mathrm{~g} \cdot \mathrm{cm}^{-3}, R(F)=0.043, R_{\mathrm{w}}\left(F^{2}\right)=0.117$.

Table 1. Parameters used for the $\mathrm{X}$-ray data collection

\begin{tabular}{ll}
\hline Crystal: & colorless needle, size $0.12 \times 0.14 \times 0.42 \mathrm{~mm}$ \\
Wavelength: & Mo $K_{\alpha}$ radiation $(0.71069 \AA)$ \\
$\mu:$ & $0.77 \mathrm{~cm}^{-1}$ \\
Diffractometer: & Enraf-Nonius CAD4 \\
Scan mode: & $\omega / 2 \theta$ \\
$T_{\text {mearunement: }}$ & $293 \mathrm{~K}$ \\
$2 \theta_{\text {max: }}$ & $45^{\circ}$ \\
N(hkl) & 1766 \\
Criterion for $I_{0}:$ & $I_{0}>2 \sigma\left(I_{0}\right)$ \\
N(param) & 208 \\
Programed: & SHELXS-86, SHELXL-93, MolEN, ZORTEP
\end{tabular}

Table 2. Final atomic coordinates and displacement parameters (in $\AA^{2}$ )

\begin{tabular}{llllll}
\hline Atom & Site & $x$ & $y$ & $z$ & $U_{\text {iso }}$ \\
\hline H(3) & $4 e$ & $0.806(6)$ & $0.391(2)$ & $-0.0178(9)$ & 0.087 \\
H(5) & $4 e$ & $0.351(6)$ & $0.106(2)$ & $-0.0228(9)$ & 0.090 \\
H(7A) & $4 e$ & $0.572(8)$ & $0.286(3)$ & $-0.105(1)$ & 0.123 \\
H(7B) & $4 e$ & $0.657(8)$ & $0.166(3)$ & $-0.097(1)$ & 0.123 \\
H(7C) & $4 e$ & $0.877(9)$ & $0.234(3)$ & $-0.089(1)$ & 0.123 \\
H(10A) & $4 e$ & $0.737(7)$ & $0.302(2)$ & $0.157(1)$ & 0.095 \\
H(10B) & $4 e$ & $0.570(7)$ & $0.180(2)$ & $0.158(1)$ & 0.095 \\
H(12) & $4 e$ & $0.430(7)$ & $0.453(2)$ & $0.200(1)$ & 0.111 \\
H(13) & $4 e$ & $0.181(9)$ & $0.503(3)$ & $0.275(1)$ & 0.141 \\
H(14) & $4 e$ & $0.027(9)$ & $0.371(3)$ & $0.329(1)$ & 0.144 \\
H(15) & $4 e$ & $0.073(9)$ & $0.182(3)$ & $0.304(1)$ & 0.139 \\
H(16) & $4 e$ & $0.312(7)$ & $0.134(3)$ & $0.228(1)$ & 0.112 \\
& & & & & \\
\hline
\end{tabular}

Table 3. Final atomic coordinates and displacement parameters (in $\AA^{2}$ )

\begin{tabular}{|c|c|c|c|c|c|c|c|c|c|c|}
\hline Atom & Site & $x$ & $y$ & $z$ & $U_{11}$ & $U_{22}$ & $U_{33}$ & $U_{12}$ & $U_{13}$ & $U_{23}$ \\
\hline$O(1)$ & $4 e$ & $0.3243(3)$ & $0.2914(1)$ & $0.11845(5)$ & $0.0476(8)$ & $0.085(1)$ & $0.0533(9)$ & $0.0106(7)$ & $0.0016(7)$ & $-0.0011(7)$ \\
\hline$N(1)$ & $4 e$ & $0.1554(5)$ & $0.1057(2)$ & $0.06585(8)$ & $0.061(1)$ & $0.073(1)$ & $0.087(1)$ & $-0.001(1)$ & $-0.001(1)$ & $-0.003(1)$ \\
\hline $\mathrm{N}(2)$ & $4 e$ & $0.7074(5)$ & $0.4437(2)$ & $0.07492(7)$ & $0.073(1)$ & $0.066(1)$ & $0.068(1)$ & $0.001(1)$ & $0.004(1)$ & $-0.001(1)$ \\
\hline$C(1)$ & $4 e$ & $0.4271(5)$ & $0.2748(2)$ & $0.07129(8)$ & $0.047(1)$ & $0.066(1)$ & $0.053(1)$ & $0.011(1)$ & $-0.001(1)$ & $0.001(1)$ \\
\hline$C(2)$ & $4 e$ & $0.6077(5)$ & $0.3514(2)$ & $0.04756(8)$ & $0.056(1)$ & $0.065(1)$ & $0.057(1)$ & $0.009(1)$ & $-0.002(1)$ & $-0.001(1)$ \\
\hline$C(3)$ & $4 e$ & $0.6903(6)$ & $0.3381(2)$ & $-0.00193(9)$ & $0.059(2)$ & $0.081(2)$ & $0.060(2)$ & $0.008(1)$ & $0.006(1)$ & $0.005(1)$ \\
\hline$C(4)$ & $4 e$ & $0.5948(6)$ & $0.2474(2)$ & $-0.03000(9)$ & $0.063(1)$ & $0.084(2)$ & $0.057(1)$ & $0.015(1)$ & $0.001(1)$ & $-0.003(1)$ \\
\hline
\end{tabular}


Table 3. (Continued)

\begin{tabular}{|c|c|c|c|c|c|c|c|c|c|c|}
\hline Atom & Site & $x$ & $y$ & $z$ & $U_{11}$ & $U_{22}$ & $U_{33}$ & $U_{12}$ & $U_{13}$ & $U_{23}$ \\
\hline$C(5)$ & $4 e$ & $0.4194(6)$ & $0.1703(2)$ & $-0.00656(9)$ & $0.060(2)$ & $0.076(2)$ & $0.070(2)$ & $0.011(1)$ & $-0.010(1)$ & $-0.013(1)$ \\
\hline$C(6)$ & $4 e$ & $0.3369(5)$ & $0.1840(2)$ & $0.04309(9)$ & $0.049(1)$ & $0.068(1)$ & $0.066(1)$ & $0.006(1)$ & $-0.002(1)$ & $-0.001(1)$ \\
\hline$C(7)$ & $4 e$ & $0.6779(9)$ & $0.2345(3)$ & $-0.0841(1)$ & $0.100(2)$ & $0.124(3)$ & $0.061(2)$ & $0.031(2)$ & $0.006(2)$ & $-0.010(2)$ \\
\hline$C(8)$ & $4 e$ & $0.0029(8)$ & $0.0441(3)$ & $0.0856(1)$ & $0.085(2)$ & $0.091(2)$ & $0.125(2)$ & $-0.013(2)$ & $0.008(2)$ & $0.002(2)$ \\
\hline$C(9)$ & $4 e$ & $0.7889(8)$ & $0.5185(3)$ & $0.0983(1)$ & $0.112(3)$ & $0.079(2)$ & $0.104(2)$ & $-0.003(2)$ & $0.003(2)$ & $-0.009(2)$ \\
\hline$C(10)$ & $4 e$ & $0.5397(6)$ & $0.2586(2)$ & $0.16064(9)$ & $0.060(2)$ & $0.102(2)$ & $0.057(1)$ & $0.016(2)$ & $-0.000(1)$ & $0.008(1)$ \\
\hline$C(11)$ & $4 e$ & $0.3925(5)$ & $0.2876(2)$ & $0.20851(8)$ & $0.046(1)$ & $0.088(2)$ & $0.053(1)$ & $0.003(1)$ & $-0.002(1)$ & $0.003(1)$ \\
\hline$C(12)$ & $4 e$ & $0.3547(7)$ & $0.3944(3)$ & $0.2222(\mathrm{~J})$ & $0.077(2)$ & $0.098(2)$ & $0.082(2)$ & $-0.005(2)$ & $0.015(2)$ & $-0.007(2)$ \\
\hline$C(13)$ & $4 e$ & $0.2187(8)$ & $0.4218(3)$ & $0.2664(1)$ & $0.100(2)$ & $0.131(3)$ & $0.096(2)$ & $0.007(2)$ & $0.013(2)$ & $-0.028(2)$ \\
\hline$C(14)$ & $4 e$ & $0.1206(8)$ & $0.3427(5)$ & $0.2968(1)$ & $0.090(2)$ & $0.172(4)$ & $0.071(2)$ & $0.003(3)$ & $0.009(2)$ & $-0.004(2)$ \\
\hline$C(15)$ & $4 e$ & $0.1511(8)$ & $0.2367(4)$ & $0.2854(1)$ & $0.093(2)$ & $0.151(4)$ & $0.078(2)$ & $-0.010(2)$ & $0.006(2)$ & $0.036(2)$ \\
\hline$C(16)$ & $4 e$ & $0.2905(7)$ & $0.2082(3)$ & $0.2402(1)$ & $0.080(2)$ & $0.104(2)$ & $0.075(2)$ & $0.004(2)$ & $0.000(2)$ & $0.013(2)$ \\
\hline
\end{tabular}

Acknowledgments. We thank the Deutsche Forschungsgemeinschaft and the Fonds der Chemischen Industrie for financial support of our work.

\section{References}

1. Ugi, I.; Fetzner, U.; Eholzer, H.; Knupfer, H.; Offerman, K.: Neuere Methoden der präparativen organischen Chemie IV, Isonitril-Synthesen. Angew. Chem. Int. Ed. Engl. 4 (1965) 472-484.

2. Hahn, F. E.; Tamm, M.; Lügger, T.: Equilibrium between Isocyanide and Carbene Complexes in Coordination Compounds of 2,6-Dihydroxyphenyl Isocyanide. Angew. Chem. Int. Ed. Engl. 33 (1994) 1356-1359 and literature quoted therein.

3. Hahn, F. E.; Tamm, M.: Equilibrium between Ylidene and Isocyanide Complexes: Synthesis and Crystal Structure of $c i s-(N$-Methyl-1,2-dihydroxybenzoxazol-2ylidene)-triphenylphosphine)tetracarbonyltungsten. Organometallics 14 (1995) 2597-2600.
4. Lentz. D.: Preugschat, D.: Structure of Pentafluorophenyl Isocyanide at 115 K. Acta Crystallogr. C49 (1993) 52-54.

5. Britton, D.; Konnert, J.: Lam, S.: 4-Bromobenzoisonitrile, $\mathrm{C}_{7} \mathrm{H}_{4} \mathrm{BrN}$, and 4-lodobenzoisonitrile, $\mathrm{C}_{7} \mathrm{H}_{4} \mathrm{IN}$. Cryst. Struct. Comm. 7 (1978) 445-448.

6. Carter, V. B.; Britton, D.; Gleason, W. B.: 2,4,6-Tribromobenzoisonitrile. $\mathrm{C}_{7} \mathrm{H}_{2} \mathrm{Br} 3 \mathrm{~N}$. Cryst. Struct. Comm. 6 (1977) 543-548.

7. Enraf-Nonius: CAD-4 Software. Version 5.0. Enraf-Nonius, Delft, The Netherlands 1989.

8. Fair, C. K.: MolEN. An Interactive Intelligent System for Crystal Structure Analysis. Enraf-Nonius, Delft, The Netherlands 1990.

9. Sheldrick, G. M.: SHELXS-86. Program for the Solution of Crystal Structures. University of Göttingen, Germany 1985.

10. Sheldrick, G. M.: SHELXL-93. Program for the Refinement of Crystal Structures. University of Göttingen, Germany 1993.

11. Zsolnai, L.; Pritzkow, H.: ZORTEP. ORTEP Program for Personalcomputer. University of Heidelberg, Germany 1995. 